\title{
Optical pulse characteristics of sonoluminescence at low acoustic drive levels
}

\author{
Vijay H. Arakeri* and Asis Giri \\ Department of Mechanical Engineering, Indian Institute of Science, Bangalore 560 012, India
}

\begin{abstract}
From a nonaqueous alkali-metal salt solution, it is possible to observe sonoluminescence (SL) at low acoustic drive levels with the ratio of the acoustic pressure amplitude to the ambient pressure being about 1 . In this case, the emission has a narrowband spectral content and consists of a few flashes of light from a levitated gas bubble going through an unstable motion. A systematic statistical study of the optical pulse characteristics of this form of SL is reported here. The results support our earlier findings [Phys. Rev. E 58, R2713 (1998)], but in addition we have clearly established a variation in the optical pulse duration with certain physical parameters such as the gas thermal conductivity. Quantitatively, the SL optical pulse width is observed to vary from $10 \mathrm{~ns}$ to $165 \mathrm{~ns}$ with the most probable value being $82 \mathrm{~ns}$, for experiments with krypton-saturated sodium salt ethylene glycol solution. With argon, the variation is similar to that of krypton but the most probable value is reduced to $62 \mathrm{~ns}$. The range is significantly smaller with helium, being from 22 ns to $65 \mathrm{~ns}$ with the most probable value also being reduced to $42 \mathrm{~ns}$. The observed large variation, for example with krypton, under otherwise fixed controllable experimental parameters indicates that it is an inherent property of the observed SL process, which is transient in nature. It is this feature that necessitated our statistical study. Numerical simulations of the SL process using the bubble dynamics approach of Kamath, Prosperetti, and Egolfopoulos [J. Acoust. Soc. Am. 94, 248 (1993)] suggest that a key uncontrolled parameter, namely the initial bubble radius, may be responsible for the observations. In spite of the fact that certain parameters in the numerical computations have to be fixed from a best fit to one set of experimental data, the observed overall experimental trends of optical pulse characteristics are predicted reasonably well.
\end{abstract}

\section{INTRODUCTION}

Cavitation is a physical process involving the formation and growth of cavities in the form of bubbles in a body of liquid due to pressure reduction. Cavities once formed subsequently collapse and this phase of the cavity motion can be quite violent, in the sense that it can lead to such extreme conditions as very high pressures and temperatures within and surrounding the cavity. This leads to some of the commonly known observable effects due to cavitation, namely material damage or erosion, generation of intense noise, and even luminescence. It is luminescence from a cavitation field with which we are presently concerned.

Depending on how the low pressures are generated, one can distinguish between two types of cavitation: hydrodynamic and acoustic. The former is associated with highspeed liquid flows as, for example, in the case of flow through a venturi. Acoustic cavitation results from pressure variations induced in the liquid by subjecting it to an intense sound or acoustic field. Luminescence is observed from both types of cavitation; however, it is the one associated with acoustic cavitation and commonly termed as "sonoluminescence (SL)', that has been studied much more extensively [1].

In light of recent developments in the field, as pointed out by Crum [2] and Matula [3], SL can primarily be classified into two types, namely multibubble SL (MBSL) and singlebubble SL (SBSL). MBSL is observed from cavitation

\footnotetext{
*Author to whom correspondence should be addressed. Email address: vijay@mecheng.iisc.ernet.in
}

bubble fields, which consist of transiently growing and collapsing cavities that are distributed randomly in the bulk of the liquid. MBSL has been the subject of extensive studies since around 1934, and the major findings from these are available in comprehensive reviews on the subject by Walton and Reynolds [1], Verral and Sehgal [4], and El'pinner [5]. One of the primary aims of the more recent studies was to obtain an estimate of what is termed "cavitation temperatures" [6]. Therefore, some gross features of MBSL have been investigated and interpreted logically on the basis of modeling the SL process using bubble dynamics formulations [7-9]. However, as it can be imagined, detailed studies such as the measurement of bubble dynamics parameters, etc., under MBSL conditions have not been possible in view of the random nature of the phenomenon. Such studies are expected to be feasible only if SL is produced under controlled conditions.

This in fact was achieved by Gaitan [10] in 1990. He was able to levitate a gas bubble in a standing-wave acoustic field and then drive it to such nonlinear motion that a flash of light was emitted during each acoustic cycle [11]. This phenomenon has come to be known as single bubble SL and its discovery has resulted in some extraordinary developments in the field; these have been reviewed by Barber et al. [12], Putterman [13], Crum [2], Putterman and Weninger [14], and Hilgenfeldt and Lohse [15]. Some of the significant findings of relevance to the present study are as follows: the SBSL flash width is in a picosecond regime [16-18], its spectrum is broadband with photon energy in excess of $6 \mathrm{eV}$ [19], and unlike MBSL, SBSL is found in a very restricted parameter space [20], but once established it is robust and can go on for hours or even longer. Many of the intriguing aspects of 
SBSL have now been satisfactorily explained on the basis of bubble dynamics formulations, and the most recent contributions in this direction are those due to Hilgenfeldt et al. [21] and Moss et al. [22].

In the above, we have considered briefly some aspects of the two types of SL, namely MBSL and SBSL; it is natural that one may examine a comparison between these two forms of SL. This has been the subject of studies by Matula et al. [23], Yasui [24], and most recently by Didenko and Gordeychuk [25]. One important difference lies in the spectrum characteristics [23]; if MBSL is generated in water with dissolved sodium chloride, its spectrum consists of not only a continuum but also prominent features that can be attributed to a band emission from $\mathrm{OH}$ radical and also a line emission from the excited sodium atoms. On the other hand, with SBSL from the same medium, its spectrum consists only of a continuum, and the absence of the abovementioned features is noticeable [23]. One similarity of relevance to the present work is the fact that the measured MBSL optical flash width is in a subnanosecond regime [26], as is the case for SBSL flash widths.

Most of the SL studies, whether MBSL or SBSL, have been associated with acoustic fields in which the ratio of the acoustic pressure amplitude, $P_{a}$, to the ambient pressure, $P_{0}$, is greater than 1 ; typical MBSL experiments involve $P_{a} / P_{0}$ greater than 2 or even larger, and typical SBSL experiments involve $P_{a} / P_{0}$ in the range of 1.2-1.5 [20]. Solutions to the bubble dynamics equation (see, for example, Hilgenfeldt et al. [27]) show that at these drive levels, the bubble motion can be highly nonlinear and in particular the bubble wall velocities during the collapse phase can definitely exceed the gas speed of sound, but also approach the liquid speed of sound, and thus we could term this type of collapse as a "hard" collapse. In the theoretical studies by Kamath et al. [28], it is shown that SL should be feasible even at a relatively low drive pressure ratio of $P_{a} / P_{0}$ being near 1 , and at these drive levels the bubble collapse is predicted to be much less violent and hence we could term this type of collapse as a "soft" collapse. In some practical applications, in particular involving sonochemistry [29], cavitation bubble fields with dominantly "soft" collapse could be desirable. Such fields may result in desired chemical products with a much better overall efficiency. Therefore, an experimental characterization of SL associated with a cavitation bubble field driven at low acoustic pressure ratios, with $P_{a} / P_{0}$ near 1 , forms the motivation for our present work. The present study in conjunction with our recent results [30] should be of interest in relation to the theoretical work of, for example, Kamath et al. [28] but also in the larger context of comparing the optical characteristics of the presently observed SL with those of MBSL and SBSL.

\section{EXPERIMENTAL APPARATUS AND METHODS}

\section{A. Acoustic resonator}

The basic apparatus used to generate sonoluminescence in the present experiments is a $250-\mathrm{ml}$ cylindrical quartz beaker. The beaker had dimensions of 70-mm OD, 2-mm thickness, and 90-mm height. After considerable trials, it was

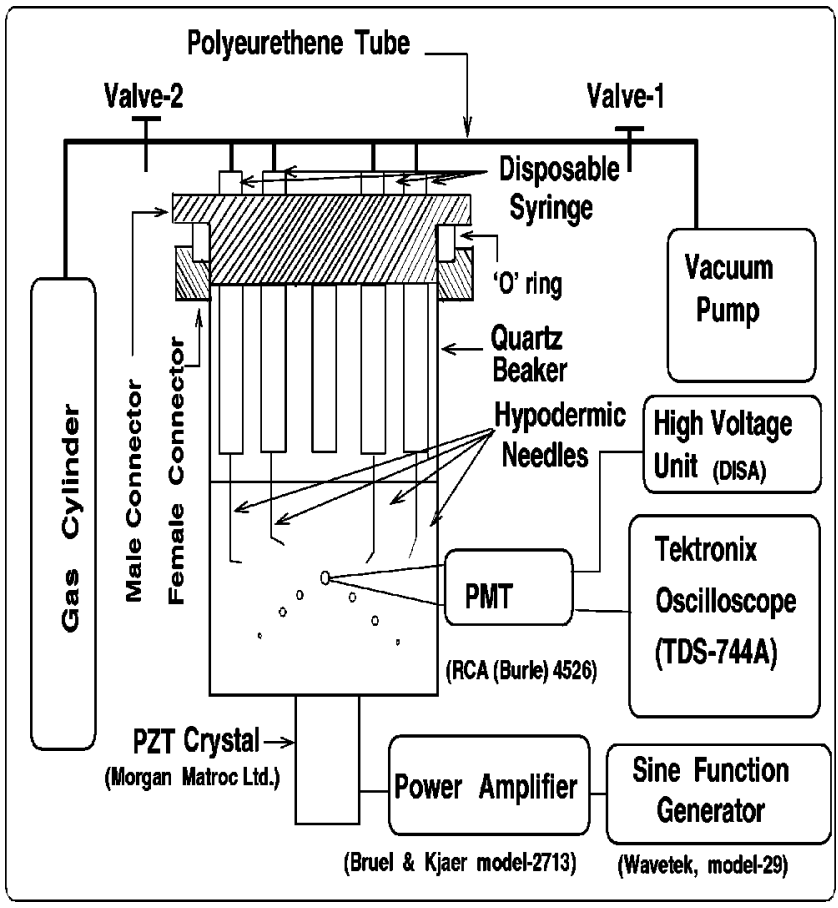

FIG. 1. A schematic of the experimental setup and instrumentation used in the present studies.

found that we got very good multibubble cavitation activity using a sample of $100 \mathrm{ml}$, thus partially filling the beaker. The beaker with the 100-ml sample was driven acoustically with a PZT cylindrical crystal (Morgan Matroc Limited) of 25.4-mm OD, 50-mm length, and 3.18-mm thickness, which was attached at the center of the bottom of the beaker. As per the data sheets supplied by the manufacturer, the fundamental resonant frequencies in length/radial modes of the crystal used are $30 \mathrm{kHz}$ and $50 \mathrm{kHz}$, respectively.

To make the beaker with the partially filled liquid sample a closed system, some modifications were necessary, and these are indicated schematically in Fig. 1. As shown in this figure, a perplex female connector is glued to the open top end of the beaker. A male connector that is basically a flat perspex circular disk is press-fitted inside the female connector with an "O' ring (68-mm ID and with a square cross section of $5 \mathrm{~mm} \times 5 \mathrm{~mm}$ ) between them. The combination of these could be termed as a "cap" and use of this made the system gas tight. The top male connector had some other fittings to enable continuous purging of the sample with the desired gas. The flat circular disk had five $6.8-\mathrm{mm}$ openings, one at the center and the other four being near the circumference of the disk. The outer four holes were connected to a gas cylinder or a vacuum pump. Each of the connecting tubes to the vacuum pump and the gas cylinder could be isolated with the help of valves that are marked as valve-1 and valve- 2 in the schematic of Fig. 1. The central hole could be used for various purposes depending on the specific requirement. For example, in one application the central hole was used for venting the gas when the liquid was purged continuously with different gases. The above-mentioned closed setup with 100-ml liquid ethylene glycol [31], which makes the liquid height in the beaker approximately $30 \mathrm{~mm}$, 
was used for the SL studies reported here. The samples were prepared in a closed setup, the details of which are presented in [32].

The whole assembly consisting of the beaker, PZT crystal, cap, and 100-ml liquid sample made a resonant acoustic system when driven at an appropriate frequency with a sine function generator (Wavetek, model-29) and a power amplifier (Brüel and Kjær, model-2713). The relevant resonant frequencies can be estimated using the method outlined by Ellis [33]. Experimentally, we worked at a frequency of about $32 \mathrm{kHz}$, which is close to the resonant frequency corresponding to the $(r=1, \theta=0, z=1)$ mode. At this frequency, we found the multibubble cavitation activity becoming close to a single bubble activity, near the center of the liquid sample, as the power level was reduced. In fact, a single bubble could be levitated at very low drive levels. At the maximum drive levels, we could obtain a maximum $P_{a}$ value of about 5 bars, and in the present work we followed the below-mentioned procedure for $P_{a}$ measurements.

First we took the gas-saturated liquid sample and established the conditions for SL at a low drive level; this activity was concentrated near the pressure antinode and we could mark its exact physical location by passing two laser beams $90^{\circ}$ apart. Then the sample was degassed in situ by subjecting it to a vacuum using the vacuum pump connected to the apparatus as indicated in the schematic of Fig. 1. If this was not done, the insertion of a pressure probe would result in the formation of a bubble at the tip and thus effect the measurements. After sufficient degassing (generally half an hour), the sample was allowed to sit for about $2 \mathrm{~h}$. Then the frequency and the drive level amplitudes were adjusted to those required for SL, and the tip of a miniature pressure transducer (PCB model-105A03, having a tip diameter of $2 \mathrm{~mm}$ with a rated rise time of $2 \mu \mathrm{sec}$ and a natural frequency of $300 \mathrm{kHz}$ ) was brought at the location of the crossing point of the two laser beams. The corresponding $P_{a}$ value reading was obtained using a calibration provided for the transducer. The same technique was used with both high and low crystal drive levels and the further details are available in [32].

\section{B. Observation of SL in a resonant system}

Before describing our experimental methods for the optical pulse width measurements, it is worthwhile to discuss the various types of SL observed in our test cell using ethylene glycol (EG) samples. It should be mentioned here that most of the previous MBSL studies have been with horn devices (see, for example, $[6,23,34,35]$ ) and all the SBSL studies have been carried out exclusively using resonant systems [11,12,17,20,36-38]. In our present experimental setup, we found the following sequence of events as the drive level was increased.

At very low drive levels (with a frequency around $32 \mathrm{kHz}$ and $P_{a}<0.2$ bar), a bubble could be seeded and levitated at the pressure antinode. As the drive level was increased, the bubble would be set into motion and eventually go into an unstable dancing mode. With a further increase in the drive level, a large number of small nuclei formed around the pressure antinode and collected to form a large unstable bubble.
This bubble, at pressure amplitudes $P_{a}$ estimated to be larger than about 0.7 bar but less than 1.0 bar, would go into a violent motion and split up into smaller bubbles as it is ejected from the pressure antinode. A new bubble then would form in its place and the cycle would repeat with an increase in the frequency as the drive level was increased from $P_{a} \sim 0.7$ bar to $P_{a} \sim 1.0$ bar. We constantly monitored whether any SL activity was associated with this type of bubble motion using a photomultiplier tube (PMT) and a high-speed digital oscilloscope. It was found that SL was associated with this type of bubble activity only when sodium salt was dissolved in ethylene glycol samples. Using the oscilloscope, we found that the SL consisted of a few flashes (generally three to four), which came synchronously with the driving sound field. As the drive level was increased further, it was found that the acoustically driven bubble/ cavitation activity would spread to many locations around the pressure antinode in the sample volume. Near the maximum drive level (as possible with the presently used power amplifier) corresponding to estimated $P_{a}$ values from 3 to 5 bars, a plume would develop from the bottom center and come around from the sides. A large number of spots in the plume and surrounding showed SL activity; we have termed this MBSL.

Our observations of SL in the gas-saturated ethylene glycol samples with $P_{a}$ values near 1 bar can be interpreted using the theoretical studies of Hilgenfeldt et al. [39] and the experimental observations of Gaitan et al. [11] and Gaitan and Holt [38]. On this basis, we can state that the SL observed presently is close to what has been described by Hilgenfeldt et al. [39] as an "unstable" SL. Typically, this form of SL occurs at a low acoustic drive amplitude when the bubble is undergoing "soft" collapse while it still contains too much gas to reach a stable diffusive equilibrium. The primary bubble pinches off microbubbles, breaks apart, and is very unstable as observed by us. The few flashes of light recorded on the oscilloscope from the unstable SL are most likely from a single bubble going through some form of instability [38-40]. It may be possible to identify the exact nature of the instability based on a high-speed motion picture recording of the phenomenon [36].

\section{Setup for measuring optical pulse width}

An overall block diagram of the instrumentation used for measuring the optical pulse widths is shown in Fig. 1. The PMT used is an RCA (Burle) 4526, which has a rated rise time of $1.7 \mathrm{~ns}$, and the maximum voltage at which it can be operated is $2000 \mathrm{~V}$. At this voltage the rated amplification is around $1.5 \times 10^{6}$ and a single photoelectron results in a maximum signal of about $-100 \mathrm{mV}$ when measured with a highspeed digitizing oscilloscope with $50-\Omega$ termination.

The heart of the instrumentation used for the optical pulse width measurements is a high-speed digitizing oscilloscope (Tektronix, model TDS-744A). In the real-time mode, using a single channel, the maximum digitizing rate possible is 2 $\mathrm{GHz}$. In the repetitive sampling mode of signal acquisition, the effective digitizing rate is significantly increased and this mode can be conveniently used for studying a repetitive phe- 


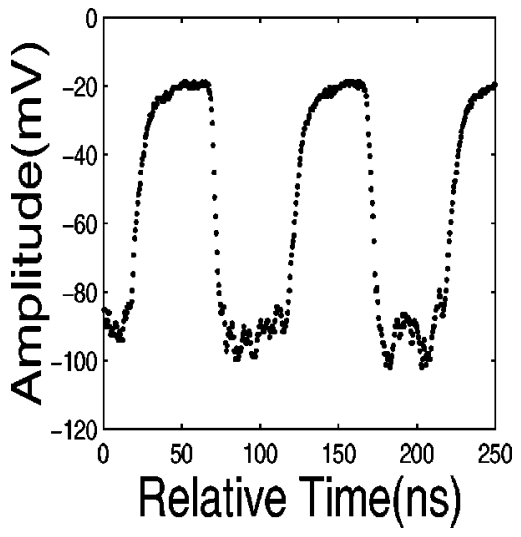

FIG. 2. The response of our optical instrumentation to pulsed 50-ns PLD light flashes. The PMT records these long-duration flashes faithfully.

nomenon like SBSL. The rated rise time and the analog bandwidth of the scope are $850 \mathrm{ps}$ and $250 \mathrm{MHz}$, respectively. The scope has many other features that are convenient for data acquisition and processing. For example, the acquired data can be stored on a floppy in various formats for further processing using a computer.

The response of our optical instrumentation to a single photoelectron event showed the rise time (defined as time for the signal to rise from $10 \%$ to $90 \%$ of the maximum amplitude) to be $1.7 \mathrm{~ns}$; as expected, this is indicative of the rise time rating of the PMT. A similar response involving a large number of photons (typical of our experiments) can be obtained by using a SBSL flash as a $\delta$-function light source. It has now been established that the optical pulse width of a SBSL flash is generally less than 340 ps [17]. It may be pointed out that the idea of using a SBSL flash as a $\delta$-function light source for the calibration of a PMT and its associated instrumentation has been previously suggested by Matula et al. [26]. We again found that the response to a SBSL flash using our instrumentation was indicative of the PMT rise time and hence the flash width was not resolved. The typical optical pulse shape corresponding to a SBSL flash is presented in [32], and it shows a rise time of $1.9 \mathrm{~ns}$. For calibration purposes, SBSL was established in the same apparatus shown schematically in Fig. 1 but using $108 \mathrm{ml}$ of degassed water.

For the system calibration to a longer duration optical pulse, we used a pulsed laser diode (PLD); a PLD will emit light as long as it is energized by a voltage pulse. In the present case, 50-ns voltage pulses were passed through the PLD. Figure 2 shows the PLD light flash signal as sensed by the presently used PMT. It is clear from Fig. 2 that our instrumentation is able to resolve longer nanosecond duration flashes faithfully. Similar calibration of the PMT response to a long duration light flash has been previously used by Matula et al. [26].

In resolving the optical pulse widths under MBSL or perhaps even an unstable SL environment, there is an additional concern that needs to be addressed. This is associated with the possibility that the observed pulse shapes are as a result of the convolution or superposition of optical signals from a large number of SL events. Matula et al. [26] have discussed this at length and have suggested certain techniques to ensure that the measured optical pulse width to a large extent corresponds to a single flash even under a MBSL environment. This involves the use of a lens and a pin hole combination ahead of the PMT to isolate spatially a small region of the test cell as seen by the PMT; presently a $5-\mathrm{cm}$ focal length lens of 30-mm diameter with a $1-\mathrm{mm}$ circular aperture assembly was used. In the case of the presently observed SL, we found virtually no difference in the pulse widths as observed with and without the additional optical components noted above. This is not surprising considering the fact that the presently investigated SL activity was limited to a small spatial zone in the neighborhood of the pressure maximum, and also most likely it originated from a single bubble going through an unstable motion. In view of this, for the present studies, we used the PMT directly.

\section{General experimental conditions}

The experiments described here were carried out using a $250-\mathrm{ml}$ quartz beaker filled with samples of $100-\mathrm{ml} \mathrm{NaCl}$ $(1.5 \mathrm{~N}, 1 \mathrm{~N}$, and $0.05 \mathrm{~N})$ salt dissolved ethylene glycol saturated with krypton, argon, and helium gases. All the rare gases had purity better than $99.99 \%$ and the tests were done with the samples being $100 \%$ saturated with the test gas. All the samples had base liquid as ethylene glycol, which has physical properties of the density, $\rho_{l}=1200 \mathrm{~kg} / \mathrm{m}^{3}$, the speed of sound, $c_{l}=1658 \mathrm{~m} / \mathrm{sec}$, the dynamic viscosity, $\mu_{l}$ $=14.8$ centipoise, and the coefficient of surface tension, $\sigma$ $=0.048 \mathrm{~N} / \mathrm{m}$. The present studies are with the acoustic drive conditions being frequency $\sim 32 \mathrm{kHz}$ and $P_{a} \sim 0.93$ bar. The environmental temperature varied from $22^{\circ} \mathrm{C}$ to $24^{\circ} \mathrm{C}$. The ambient pressure at the location where the experiments have been carried out is $\simeq 0.9$ bar.

\section{EXPERIMENTAL RESULTS}

The current study primarily involves the optical pulse characterization of SL flashes whose spectrum is a narrowband line emission in the form of sodium-D line resonance radiation. This narrowband line emission for each set of experimental conditions was confirmed by using a scanning monochromator in the wavelength range of $300 \mathrm{~nm}$ to 700 $\mathrm{nm}[32]$.

\section{A. Optical pulse characteristics}

In our previous article [30], we showed evidence of large nanosecond optical pulses associated with SL in the form of narrowband line emission. These magnitudes being in tens of nanoseconds are certainly resolvable using our optical instrumentation, whose response time as determined using a SBSL flash is $\sim 1.9$ ns. However, from the observation of the oscilloscope traces, it was clear that there were considerable variations in the pulse characteristics in terms of the pulse amplitude and duration from one pulse to another [41]. As a result, any meaningful conclusions could be arrived at only on the basis of a statistical study. For this using the built-in facility in the oscilloscope, 800 individual optical pulses 
were acquired and stored in 1.44-MB floppies for later analysis using a computer. The acquisition and storing of each optical pulse on the floppy took almost half a minute; thus in $1 \mathrm{~h}$ we could acquire and store about 120 pulses. Taking 800 pulses consumed nearly $6.5 \mathrm{~h}$. Over this long duration, care had to be exercised to keep the experimental conditions constant; in particular, a continuous purging with the cover gas was required. In addition, we restricted our statistical study to the SL regime with a low acoustic drive level, since in this case as indicated earlier we were fairly certain that the PMT signal was not distorted due to the convolution or superposition of optical signals from a large number of SL events. Another point needs to be mentioned here: for acquiring a PMT signal, we had to set a trigger level on the digitizing oscilloscope. If this was set at too low a level, such as below $-40 \mathrm{mV}$ (frequently encountered single photoelectron signal level), then these pulses would often appear and a statistical study was difficult. Similarly, if it was set at a value such as $-60 \mathrm{mV}$, SL pulses were recorded, but they were highly distorted and a meaningful Gaussian fit was difficult. Therefore, all the statistical study results to be presented refer to when the trigger level was set at $-110 \mathrm{mV}$. Each set of data stored in a floppy was transferred to a computer for further processing. A Gaussian best fit was done as detailed elsewhere [26,32]. From this fit we obtained the data on the pulse width [full width at half maximum (FWHM)] in ns and the pulse area in nVs. Below we provide the results of our statistical study.

\section{B. Optical pulse statistics}

\section{Pulse width}

Figure 3 shows the statistical distribution of the SL optical pulse widths for 1-N sodium chloride glycol solution saturated with helium, argon, and krypton gases. From these figures, it can be noted that the pulse widths are large and confirm the existence of nanosecond pulse duration. Further, we see that for different gases, the peak in the distribution occurs at different pulse width regions; for krypton, it lies in a broad range of around 65-110 ns, in the case of argon it is concentrated in the range of around 50-65 ns, whereas in the case of helium it is limited to around $42 \mathrm{~ns}$. In addition, the overall ranges of the pulse widths observed are different for different gases. In the case of krypton and argon saturated salt solutions, the pulse widths vary from $10 \mathrm{~ns}$ to as high as $165 \mathrm{~ns}$, whereas in the case of helium they vary only in the range from $22 \mathrm{~ns}$ to $70 \mathrm{~ns}$. Such a large variation of the observed pulse widths, in particular for krypton- and argonsaturated solutions, is perhaps indicative of the sensitivity of the SL process to some parameters; an attempt to identify some of these will be made later on the basis of numerical computations. From the above results, it can also be pointed out that there are definite indications that the pulse width decreases with an increase in the gas thermal conductivity. Figure 4 shows the statistics of the optical pulse widths for the presently observed SL with $0.05 \mathrm{~N}, 1 \mathrm{~N}$, and $1.5 \mathrm{~N}$ sodium chloride argon-saturated solutions. The change in the normality does not seem to effect the optical pulse width distributions in a significant way. However, we found that
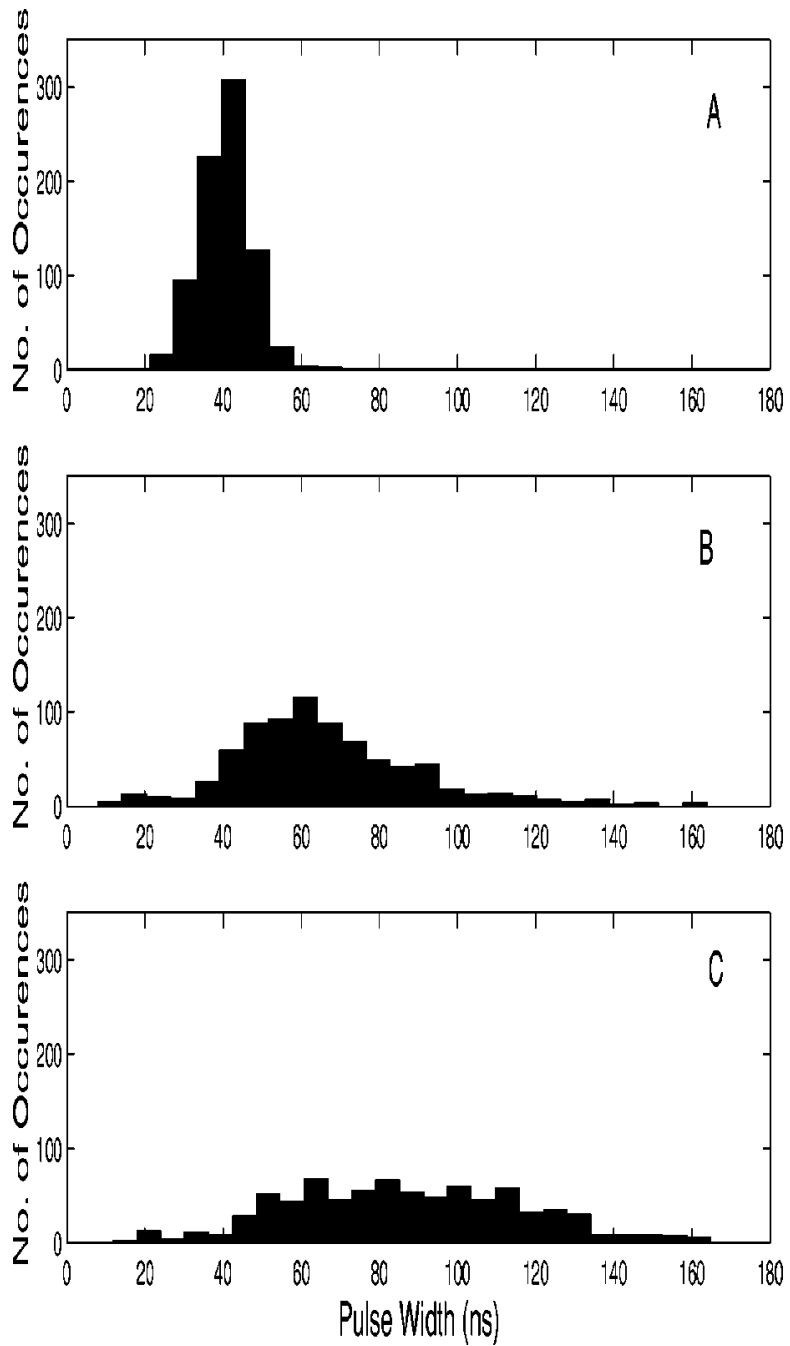

FIG. 3. The distribution of the optical pulse widths (at FWHM) for SL generated from 1-N sodium chloride dissolved ethylene glycol (NaCl/EG) solution saturated with (A) helium, (B) argon, and (C) krypton gases; acoustic pressure amplitude $P_{a} \sim 0.93$ bar and a drive frequency $f_{a} \sim 32 \mathrm{kHz}$.

the frequency of occurrence of SL flashes reduced with a decrease in the normality, especially when $0.05 \mathrm{~N}$ was used.

\section{Pulse area}

Figure 5 shows the statistical distributions of a SL optical pulse area for 1-N sodium chloride glycol solution saturated with helium, argon, and krypton gases, and similar results for different normality with argon gas are shown in Fig. 6. It is known that the pulse area is directly proportional to the number of photons emitted per flash, and this number seems to depend on the type of dissolved gas in the solution and it decreases in the order of decreasing atomic weight of the gas. This can be inferred from comparing the results presented in Figs. 5(A), 5(B), and 5(C).

\section{DISCUSSION OF RESULTS}

\section{A. Optical pulse characteristics}

All the results presented here on the SL optical pulse characteristics are with sodium chloride salt dissolved ethyl- 

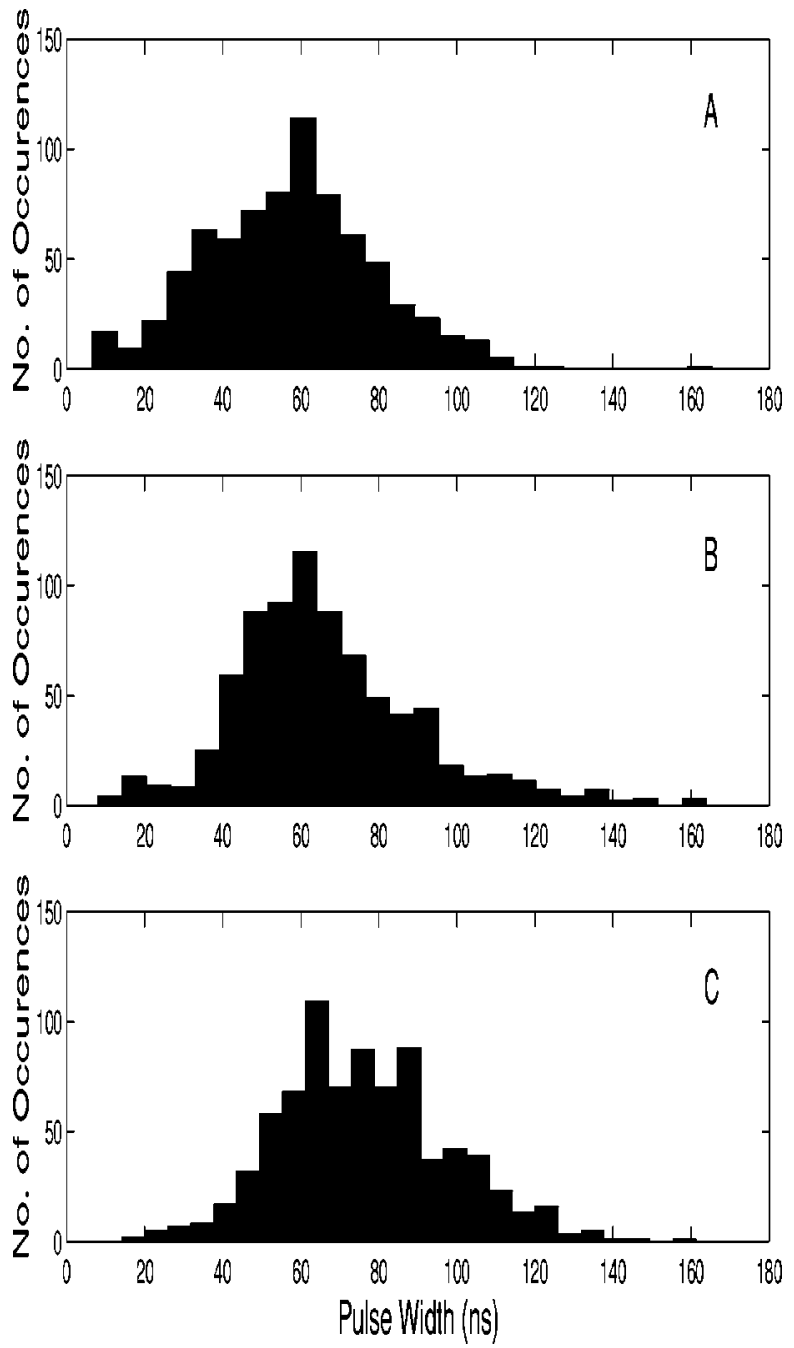

FIG. 4. The distribution of the optical pulse widths for SL generated from argon-saturated (A) $0.05-\mathrm{N},(\mathrm{B}) 1-\mathrm{N}$, and (C) $1.5-\mathrm{N}$ $\mathrm{NaCl} / \mathrm{EG}$ solutions; $P_{a} \sim 0.93$ bar and $f_{a} \sim 32 \mathrm{kHz}$.

ene glycol solutions at low acoustic drive levels where unstable SL is observed. As pointed out earlier, the observed optical pulse duration are relatively large and they are of the order of nanoseconds and differ considerably from those observed for SBSL $[17,18]$. We can perhaps explain the difference on the basis of considering the bubble dynamics behavior with a varying pressure amplitude. As indicated earlier, the relative acoustic pressure amplitudes $\left(P_{a} / P_{0}\right)$ used presently are near 1 , whereas those for SBSL are in the range of 1.2-1.5. From the theoretical studies of Hilgenfeldt et al. [27], we expect the bubble dynamics behavior to be quite different for $P_{a} / P_{0}$ values around 1 and those greater than 1.2; the collapse is predicted to be much more violent in the latter case. Further, in the present case, the viscosity of the liquid used is 15 times higher than that of water, and this will have an added effect on the overall dynamics of a bubble. This is the case for a fixed $R_{0}$; but from the phase-space analysis by Hilgenfeldt et al. [39], for a given gas saturation the magnitude of $R_{0}$ itself will change with the $P_{a} / P_{0}$ value. Therefore, on the whole, we may expect the range of time scales, a measure of which can be estimated [21] by consid-
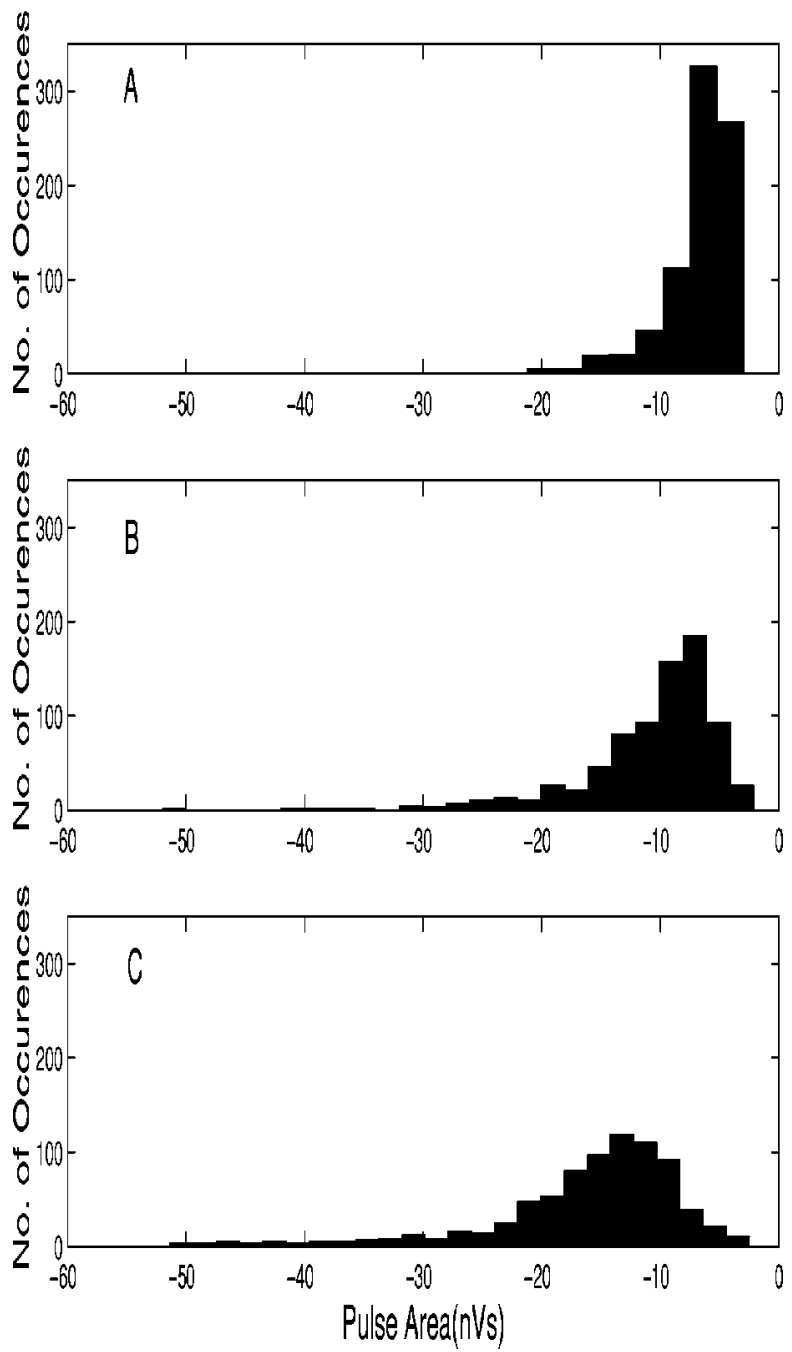

FIG. 5. The distribution of the optical pulse area for SL generated from 1-N NaCl/EG solution saturated with (A) helium, (B) argon, and (C) krypton gases; $P_{a} \sim 0.93$ bar and $f_{a} \sim 32 \mathrm{kHz}$.

ering the ratio of the instantaneous bubble radius to the wall velocity $R / \dot{R}$ during the collapse phase, to be quite different under the present conditions as compared to those for SBSL. This will have a direct bearing on the time duration for which the temperatures remain high inside the bubble and thus influence the optical pulse widths. Therefore, we believe our observations are consistent with the recent theoretical formulations $[21,27]$.

Our findings are highlighted in summary form in Table I and we see a systematic effect both on the pulse width and the number of photons emitted per flash when the dissolved gas is changed. This can also be inferred by comparing the results in Figs. 3(A), 3(B), and 3(C) for the pulse width and Figs. 5(A), 5(B), and 5(C) for the pulse area. As seen from Fig. 3, the pulse width distribution becomes broadened as we go from $\mathrm{He}$ to Ar to $\mathrm{Kr}$. Also, the pulse width where the number of occurrences peaks, which we may term as the "most probable pulse width,' shifts to a larger value, with the largest value ( $\sim 82 \mathrm{~ns}$ ) being for krypton and the smallest value $(\sim 42 \mathrm{~ns})$ being for helium. Similarly, the pulse area at which the number of occurrences is maximum shifts 

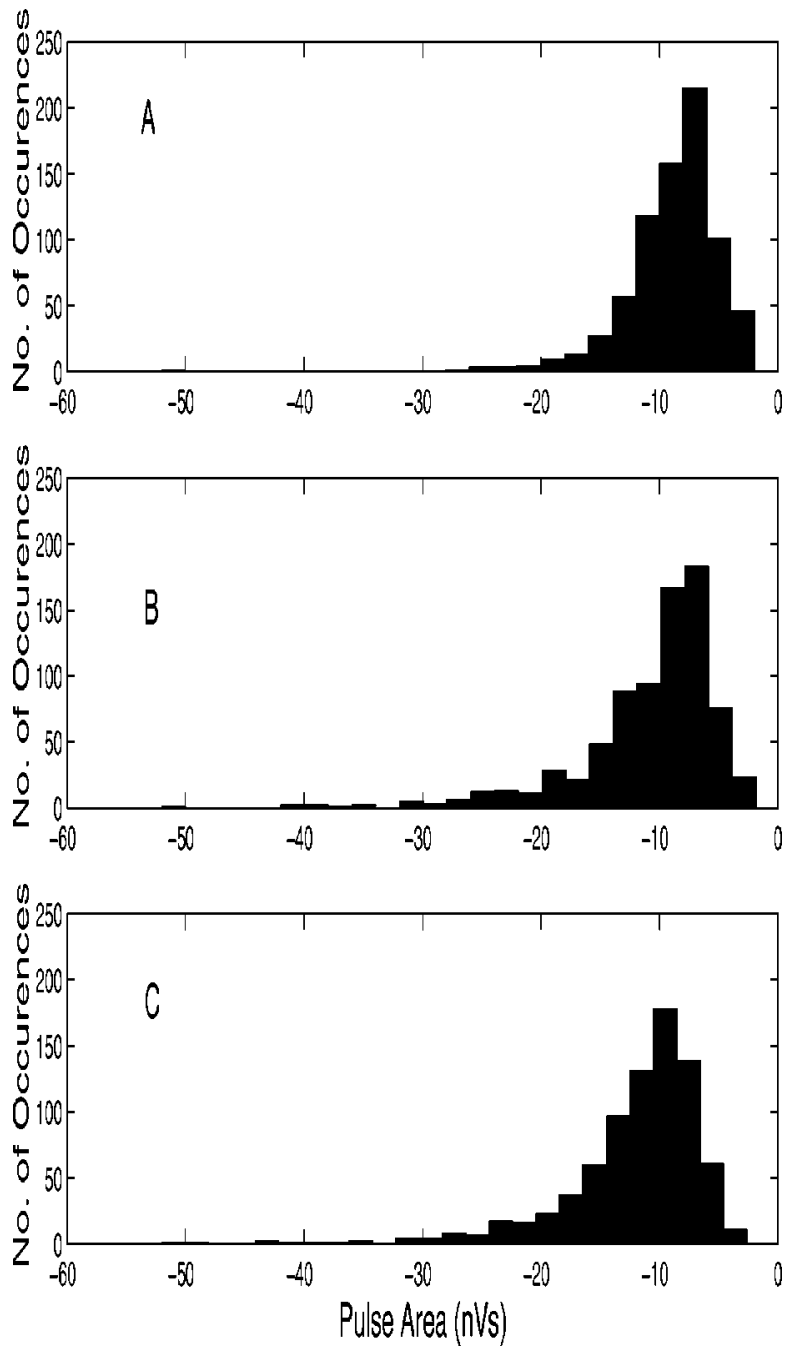

FIG. 6. The distribution of the optical pulse area for SL generated from argon-saturated (A) $0.05-\mathrm{N}$, (B) $1-\mathrm{N}$, and (C) $1.5-\mathrm{N}$ $\mathrm{NaCl} / \mathrm{EG}$ solutions; $P_{a} \sim 0.93$ bar and $f_{a} \sim 32 \mathrm{kHz}$.

to a larger value as we go up the atomic weight of the noble gas. In addition to the rare gases, we have carried out a few tests with the use of $\mathrm{N}_{2}$ saturated salt solution [42]; since the $\gamma$ value ( $\gamma=C_{p} / C_{v}$, the ratio of heat capacities) for $\mathrm{N}_{2}$ is lower (also it varies with the temperature), we should see a noticeable effect on the optical pulse characteristics. This indeed is true; as indicated in Table I with $\mathrm{N}_{2}$ both the optical pulse width and area are significantly lower than those for the rare gases. It was suggested earlier that the dependence of the type noted in Table I for the rare gases may be related to the thermal conductivity of the gas in question. This point will be considered later when we compare the experimentally observed findings with the predictions from numerical computations of the governing bubble dynamics equations.

Another parameter that was varied in our experiments but had a rather small effect on the results was the normality of the solution. Comparative results for different normality are presented in Fig. 4 for the pulse width and Fig. 6 for the pulse area. Even though we may not expect a significant effect on the pulse width distribution with normality, the same should not be the case with the pulse area, since it is directly related to the intensity of SL flashes. With an increase in the normality, the number of sodium atoms within the bubble is expected to increase and hence this should reflect as an increase in the SL intensity. However, this does not seem to be the case, since as shown in Figs. 6(A) and $6(\mathrm{C})$, the pulse area distributions seem to be very similar, both quantitatively and qualitatively, even with a change of normality from $0.05 \mathrm{~N}$ to $1.5 \mathrm{~N}$, an increase by a factor of 30. This may be an indication of the intensity saturation effect as found by Flint and Suslick [35] with an increase in the normality of a potassium salt. An explanation for this may not be easily forthcoming, but the fact that the saturation solubility value for argon gas may depend on the normality and thus have a subtle effect on the bubble dynamics could be one reason. Some evidence to the increase in the dissolved gas content with an increase in the normality was present in our experiments.

\section{B. Comparison of experimental observations with computations}

In our previous article [30], we proposed a model to explain the large optical pulse widths observed in experiments

TABLE I. A summary of the experimental findings. EG denotes ethylene glycol.

\begin{tabular}{|c|c|c|c|c|c|}
\hline Liquid used & $\begin{array}{l}\text { Gas } \\
\text { used }\end{array}$ & $\begin{array}{l}\text { Most probable value } \\
\text { of pulse width }\end{array}$ & $\begin{array}{l}\text { Maximum value } \\
\text { of pulse area }\end{array}$ & $\begin{array}{l}\text { Maximum number } \\
\text { of photons/pulse }\end{array}$ & $\begin{array}{c}\text { Most probable value } \\
\text { of photon/pulse }\end{array}$ \\
\hline $\mathrm{EG}+1 \mathrm{~N} \mathrm{NaCl}$ & $\mathrm{Kr}$ & $82 \mathrm{~ns}$ & $-51 \mathrm{nVs}$ & $6.06 \times 10^{6}$ & $1.48 \times 10^{6}$ \\
\hline $\mathrm{EG}+1 \mathrm{~N} \mathrm{NaCl}$ & $\mathrm{Ar}$ & $62 \mathrm{~ns}$ & $-44 \mathrm{nVs}$ & $5.23 \times 10^{6}$ & $1.05 \times 10^{6}$ \\
\hline $\mathrm{EG}+1 \mathrm{~N} \mathrm{NaCl}$ & $\mathrm{He}$ & $42 \mathrm{~ns}$ & $-26 \mathrm{nVs}$ & $3.09 \times 10^{6}$ & $0.69 \times 10^{6}$ \\
\hline $\mathrm{EG}+1 \mathrm{~N} \mathrm{NaCl}$ & $\mathrm{N}_{2}$ & $33 \mathrm{~ns}$ & $-13.2 \mathrm{nVs}$ & $1.57 \times 10^{5}$ & $0.52 \times 10^{6}$ \\
\hline $\mathrm{EG}+0.05 \mathrm{~N} \mathrm{NaCl}$ & $\mathrm{Ar}$ & $60 \mathrm{~ns}$ & $-26 \mathrm{nVs}$ & $3.09 \times 10^{6}$ & $1.00 \times 10^{6}$ \\
\hline $\mathrm{EG}+1.5 \mathrm{~N} \mathrm{NaCl}$ & $\mathrm{Ar}$ & $62 \mathrm{~ns}$ & $-48 \mathrm{nVs}$ & $5.70 \times 10^{6}$ & $1.10 \times 10^{6}$ \\
\hline
\end{tabular}


similar to the present ones. The basis for this is what we have termed "soft" bubble collapse analyzed by Kamath et al. [28]. Here we use the mathematical formulation of Prosperetti et al. [43] to numerically compute the temperature distribution within the bubble for our experimental conditions and then, assuming thermal excitation for the sodium atoms, an estimate for the intensity of sodium-D line emission is made; hence, some quantitative comparisons with observations will be possible. Below, we provide the mathematical formulation and the results, but the details of the numerical schemes employed for the actual computations are provided in [32].

\section{Mathematical formulation}

\section{Bubble dynamics equation}

A useful form of equation describing the radial dynamics of a spherical bubble accounting liquid compressibility to first order was given by Keller and Miksis [44],

$$
\begin{aligned}
\left(1-\frac{\dot{R}}{c_{l}}\right) R \ddot{R}+\frac{3}{2}\left(1-\frac{\dot{R}}{3 c_{l}}\right) \dot{R}^{2} \\
=\frac{1}{\rho_{l}}\left(1+\frac{\dot{R}}{c_{l}}+\frac{R}{c_{l}} \frac{d}{d t}\right) \times\left[p_{l}-P_{s}(t)-P_{0}\right] .
\end{aligned}
$$

In Eq. (1), $R$ is the instantaneous bubble radius, dot denotes the time derivative, $c_{l}$ is the liquid speed of sound, $P_{0}$ is the ambient static pressure, $P_{s}(t)$ is the imposed sound or acoustic field at the location of the bubble, and $p_{l}$ is the pressure on the liquid side of the bubble interface, which is related to the bubble internal pressure $p_{g}$ through $p_{l}=p_{g}$ $-(2 \sigma / R)-(4 \mu \dot{R} / R)$; here, $\sigma$ is the surface tension coefficient and $\mu$ is the liquid viscosity. In this study, we take the sound field to be sinusoidal; therefore, $P_{s}(t)=P_{a} \cos (w t)$ with $P_{a}$ being the acoustic pressure amplitude and $w$ the driving angular frequency. The standard initial conditions used for the solution of Eq. (1) are $R(t=0)=R_{0}$ and $\dot{R}(t$ $=0)=0$.

\section{Equations for the bubble interior}

The motion of the gas inside the bubble is described by the conservation of mass, momentum, and energy equations and the development used here is from Prosperetti et al. [43]. In the formulation, the mass transfer across the bubble wall is neglected considering the diffusion time scale being much larger than the time scale of the imposed acoustic field. In addition, it is assumed that the bubble collapse is spherically symmetric due to the low acoustic pressure amplitude used; however, this point needs further consideration and will be taken up later. Another important simplifying assumption made is that the internal gas pressure is considered to be spatially uniform. In addition, the contribution of the viscous term in the momentum equation is expected to be small. Quantitative estimates to justify the latter two assumptions are provided in Prosperetti et al. [43]. Thus with these assumptions, there is no need to solve the momentum equation, and the combination of the conservation equations of mass and energy result in the following expressions (see Prosperetti et al. [43]) for computing the pressure $p_{g}(t)$ and the temperature $T(r, t)$ within the bubble:

$$
\dot{p}_{g}=\frac{3}{R}\left(\left.(\gamma-1) K \frac{\partial T}{\partial r}\right|_{r=0}-\gamma p_{g} \dot{R}\right)
$$

and

$$
\frac{\gamma}{\gamma-1} \frac{p_{g}}{T}\left[\frac{\partial T}{\partial t}+\left(\frac{\gamma-1}{\gamma p_{g}} K \frac{\partial T}{\partial r}-\frac{r \dot{p}_{g}}{3 \gamma p_{g}}\right) \frac{\partial T}{\partial r}\right]-\dot{p}_{g}=\nabla \cdot K \nabla T .
$$

Here, $\gamma\left(=C_{P} / C_{v}\right)$ is the ratio of heat capacities and assumed to be constant; $K$ is the thermal conductivity of the gas and is taken to depend linearly on the temperature. Appropriate boundary conditions are needed to solve Eq. (3). Because of the symmetry at the center of the bubble, the temperature gradient is considered to be zero, i.e., at $r=0$, $\partial T / \partial r=0$, and the bubble wall is assumed to be at the bulk liquid temperature, i.e., at $r=R, T=T_{\infty}$ [45].

As mentioned earlier, in Eq. (3) the thermal conductivity variation is considered and it is assumed to follow a linear dependence on $T$ and is expressed as

$$
K=A T+B .
$$

The values of $A$ and $B$ depend on the gas considered. We have used for argon $A=3.2 \times 10^{-5} \mathrm{~W} / \mathrm{m} \mathrm{K}^{2}, \quad B$ $=0.009 \mathrm{~W} / \mathrm{m} \mathrm{K}$ (see Cook [46]); for krypton, $A=1.54$ $\times 10^{-5} \mathrm{~W} / \mathrm{m} \mathrm{K}^{2}, B=0.0047 \mathrm{~W} / \mathrm{m} \mathrm{K}$ (see Collins and Menard [47]) and for helium, $A=30.5 \times 10^{-5} \mathrm{~W} / \mathrm{m} \mathrm{K}^{2}, \quad B$ $=0.05909 \mathrm{~W} / \mathrm{m} \mathrm{K}$ (see Collins et al. [48]). The above constants generally give good agreement with the experimentally measured values in the temperature range of $300 \mathrm{~K}$ to about $2500 \mathrm{~K}$. We assumed the thermal conductivity of the bubble contents to be dominated by the rare gases considered. This is on the basis of low vapor pressure of ethylene glycol $(\sim 0.009$ Torr) and our estimated mole fraction of the sodium atoms in the bubble from a best fit to one set of experimental results being 0.01 . Another point to be noted is that, at low acoustic drive levels considered by us, the bubble volume expansion ratio (initial volume/maximum volume) is only about $1 / 10$ (see Fig. 7) and thus the internal pressure should not go below the liquid vapor pressure and this should preclude any vaporization during the expansion phase. Therefore, we do not expect the physical properties of the bubble contents to be significantly effected by the presence of ethylene glycol vapor and sodium atoms.

The mathematical formulation presently used and indicated above assumes a spherical bubble behavior. However, in the physical description of the presently observed SL in Sec. II B, we pointed out that the optical emission coincided with an unstable behavior of the levitated bubble, leading to its eventual fragmentation. As pointed out by Matula et al. [23], such unstable bubbles may not collapse spherically; however, the contents of the bubble can still be subject to heating, but it may not be as drastic as in the case of SBSL. Therefore, our modeling does involve an approximation and 


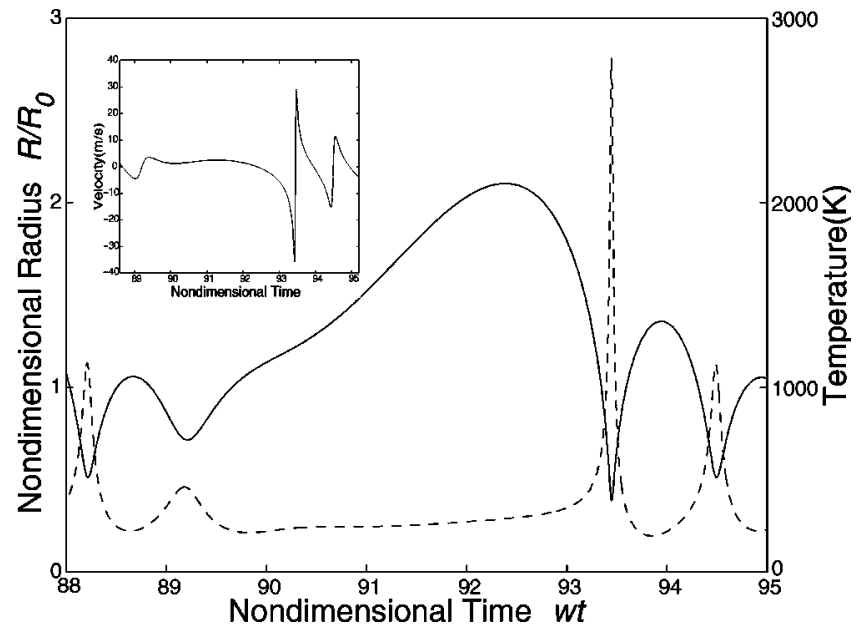

FIG. 7. The computed variation of the normalized bubble radius (solid line) and the bubble center temperature (dashed line) as a function of normalized time for $22-\mu \mathrm{m}$ argon bubble in glycol during the 15th cycle of bubble motion. Inset shows the bubble wall velocity as a function of nondimensional time for the 15 th cycle of bubble motion; $R_{0}=22 \mu \mathrm{m}, P_{a}=0.93 \mathrm{bar}$, and $f_{a}=32 \mathrm{kHz}$.

it is made for the sake of simplicity and with the expectation that the essential aspects of the phenomenon will be captured adequately.

\section{Sodium-D line intensity}

The above bubble dynamics formulation as used presently is totally consistent with that of Prosperetti et al. [43]; however, the following development on the estimation of the sodium line intensity is our own. To obtain the sodium-D line intensity using the calculated temperature field, an expression for computing the number of excited sodium atoms is required. If thermal equilibrium is assumed to be established in the bubble [49], then statistical mechanics yields the following relationship between the number $n_{1}^{*}$ of $\mathrm{Na}$ atoms per $\mathrm{cm}^{3}$ in the excited level $q$ and the total number $n_{1}$ of $\mathrm{Na}$ atoms per $\mathrm{cm}^{3}$ :

$$
n_{1}^{*}=n_{1} \frac{g_{q}}{Z} \exp \left(-\frac{E_{q}}{k T}\right)
$$

This is the Boltzmann equation in which $E_{q}$ is the excitation energy, $k$ is the Boltzmann constant, $T$ is the absolute temperature, $g_{q}$ is the statistical weight of the excited level, and $Z$ is the partition function or state sum. In general, $Z$ is given by a summation taken over all the possible energy levels, including the ground level $(q=0)$. However, as pointed out in [50], for the sodium-D line we can to a very good approximation take $g_{q} / Z=2$ and also use $E_{q}=2.1$ in Eq. (5). The rate of photon emission $\left(d N_{p} /\left.d t\right|_{b}\right)$ from a small elemental volume $d V$ within the bubble, over a solid angle of $4 \pi$ and over the whole width of the spectral line of central frequency $\nu_{0}$, is given by

$$
\left.\frac{d N_{p}}{d t}\right|_{b}=2 A_{1} n_{1} \exp \left(-\frac{2.1}{k T}\right) d V .
$$

Here, $A_{1}$ is the Einstein transition probability for spontaneous emission. With strong resonance lines, some of the emitted photons are "lost" through self-absorption; however, from the measured spectra there is no evidence for this in the present situation [30,32]. Therefore, for computing $d N_{p} /\left.d t\right|_{b}$ from the whole bubble, Eq. (6) can be integrated over the bubble volume or

$$
\left.\frac{d N_{p}}{d t}\right|_{b}=2 A_{1} n_{1} \int_{0}^{R} \exp \left(-\frac{2.1}{k T}\right) 4 \pi r^{2} d r
$$

Introducing a normalized coordinate $y=r / R$, where $r$ is a point within the bubble and $R$ is the instantaneous bubble radius, Eq. (7) becomes

$$
\left.\frac{d N_{p}}{d t}\right|_{b}=2 A_{1} n_{1}\left(4 \pi R^{3}\right) \int_{0}^{1} \exp \left(-\frac{2.1}{k T}\right) y^{2} d y .
$$

Now we expect that the number of sodium atoms initially in the bubble will remain the same throughout its life cycle and hence we can set $n_{1} \frac{4}{3} \pi R^{3}=n_{0}$, where $n_{0}$ is a constant equal to the initial number of sodium atoms in the bubble. Therefore, Eq. (8) can be written as

$$
\left.\frac{d N_{p}}{d t}\right|_{b}=6 A_{1} n_{0} \int_{0}^{1} \exp \left(-\frac{2.1}{k T}\right) y^{2} d y
$$

\section{Synthetic optical pulse}

Once the temperature field within the bubble is computed, the corresponding photon emission rate at any given time of bubble motion can be obtained from using Eq. (9). We can ask if this rate is exposed to a PMT, what would be its response, that is, can we construct a synthetic PMT response pulse from computation? This is indeed possible as described below. The number of photons $N_{p}$ is related to the charge collected by the PMT through the following relationship [51]:

$$
N_{p}=\frac{Q}{Q_{E} \times G_{\mathrm{PMT}} \times(\text { charge of electron })} \times \frac{4 \pi}{\Omega} .
$$

Here, $Q$ is the charge, $Q_{E}$ is the quantum efficiency, $G_{\mathrm{PMT}}$ is the PMT gain, and $\Omega$ is the solid angle over which the photons are collected. By differentiating Eq. (10), we obtain

$$
\left.\frac{d N_{p}}{d t}\right|_{b}=\frac{d Q}{d t} \times \frac{4 \pi}{Q_{E} \times G_{\mathrm{PMT}} \times(\text { charge of electron }) \times \Omega}
$$

in which $d Q / d t=i=V / R_{l}$, with $R_{l}$ being the load resistance. Therefore, from the computed $d N_{p} /\left.d t\right|_{b}$, the corresponding PMT signal or the synthetic optical pulse shape is given by 


$$
\begin{aligned}
V(t)= & -R_{l} \times G_{\mathrm{PMT}} \times Q_{E} \times(\text { charge of electron }) \times \frac{\Omega}{4 \pi} \\
& \times\left.\frac{d N_{p}}{d t}\right|_{b} .
\end{aligned}
$$

We have inserted a negative sign to take care of the response characteristic of a normal PMT. To compare the synthetic optical pulse with an actually observed one (corresponding to our experimental conditions), we have used the following numerical values for the constants appearing in Eqs. (9) and (12): $R_{l}=50 \Omega, \Omega / 4 \pi=\frac{1}{208}, Q_{E}$ $=0.17, G_{\mathrm{PMT}}=1.5 \times 10^{6}$, ( charge of electron $)=1.6 \times 10^{-19}$ Coulomb/electron, and $A_{1}=0.622 \times 10^{8}$ per sec.

\section{Numerical results}

The numerical computations involve the following steps. Equations (1), (2), and (3) are solved simultaneously for obtaining $R(t), p_{g}(t)$ and $T(r, t)$ or $T(y, t)$. The details of the computational schemes used presently can be seen elsewhere $[32,43]$. Once $T(y, t)$ is known, then the synthetic optical pulse can be generated using Eqs. (9) and (12); however, there is one important unknown parameter, namely $n_{0}$. The value of $n_{0}$ is fixed by a best fit to one set of experimental data. For the acoustic pressure amplitude $P_{a}$, we have used the value corresponding to most of our experimental observations, namely 0.93 bar. However, to investigate the effect of this parameter on the solutions, limited computations were made with $P_{a}$ values of 1 and 1.1 bar. The initial bubble radius $R_{0}$ is an unknown. Following [28], we have scanned a region of $0.135<R_{0} / R_{\text {res }}<0.2708$. In the present case, the value of the resonance radius, $R_{\text {res }}$, is $96 \mu \mathrm{m}$. Most of the computational results are presented with an initial bubble radius of $22 \mu \mathrm{m}$ since this appears to give the best agreement with one set of experimental observations. Then keeping $R_{0}$ and $n_{0}$ fixed, we have examined the effect of other variables such as the gas thermal conductivity.

In Fig. 7, we show (solid line) the nondimensional bubble radius $\left(R / R_{0}\right)$ as a function of nondimensional time for the 15 th cycle of bubble motion for an initial bubble radius of $22 \mu \mathrm{m}$. During the rarefaction part of the sound field, the bubble expands to a maximum radius of 2.1 times its initial radius; then the bubble collapses rapidly to a minimum radius of $0.39 R_{0}$. Eventually, the pressure builts up inside the bubble, which arrests the further inward motion of the bubble. As a result, the bubble bounces back from its minimum radius and goes through an oscillatory motion. The maximum velocity (see the inset in Fig. 7) reached during the collapse phase is about $40 \mathrm{~m} / \mathrm{s}$, which is much lower than either the gas or the liquid speed of sound. So, from this consideration our terming of this type of collapse as a "soft", collapse seems to be justifiable.

The center temperature variation as a function of nondimensional time during the 15 th cycle of bubble motion is also shown in Fig. 7 (dashed line). At the maximum radius, the bubble center temperature attains a value of around 288 $\mathrm{K}$, which is very close to the liquid temperature. From this, it can be inferred that during the expansion phase, the bubble

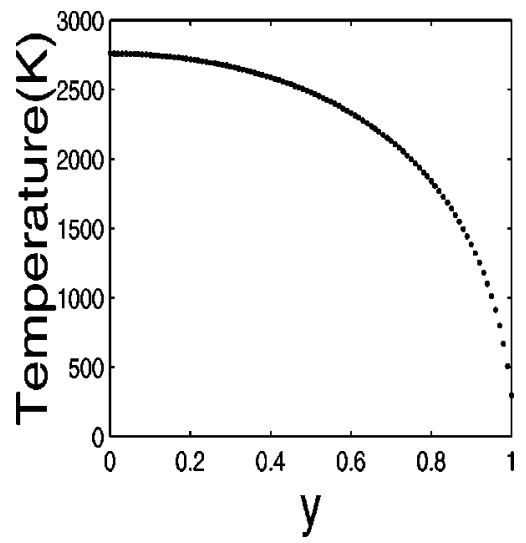

FIG. 8. The variation of the gas temperature as a function of $y$ $=r / R$ at the instant of minimum bubble radius for a $22-\mu \mathrm{m}$ argon bubble in glycol during the 15th cycle of bubble motion; $P_{a}$ $=0.93$ bar and $f_{a}=32 \mathrm{kHz}$.

behaves almost isothermally and this is now a wellestablished fact. During the end of the collapse phase, i.e., at the minimum radius, the bubble center temperature is predicted to reach a value of around $2760 \mathrm{~K}$. The temperature distribution within the bubble under this condition is presented in Fig. 8. These findings are very similar to those by Kamath et al. [28] for the case of a 26- $\mu \mathrm{m}$ argon bubble in water driven at an acoustic pressure amplitude of 0.93 bar.

In Fig. 9, we show a comparison of a synthetic optical pulse with one set of experimental data; the best fit is obtained with an $n_{0}$ value of around $1.1 \times 10^{10}$ (it may be noted that this corresponds to a density of $2.5 \times 10^{17}$ atoms per $\mathrm{cm}^{3}$ for the initial bubble radius of $22 \mu \mathrm{m}$ and this is in good agreement with a suggested value by Taylor and Jarman [52]). The general shape of the synthetic optical pulse is in very good agreement with a mean line passing through the data. The optical pulse width (at FWHM) for the results shown in Fig. 9 is about 62 ns, being the most probable value for argon (see Table I), and this corresponds to an assumed

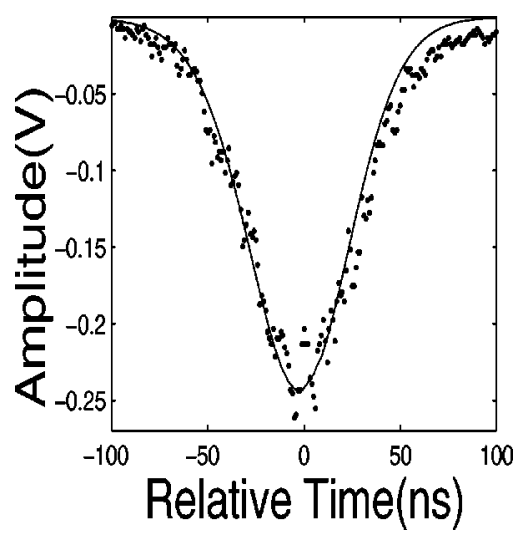

FIG. 9. The synthetic optical pulse (solid curve) as a function of relative time for a $22-\mu \mathrm{m}$ argon bubble in glycol driven at 0.93 bar acoustic pressure amplitude and a frequency of $32 \mathrm{kHz}$. The dotted points are data acquired from an experiment with a sample of argon-saturated 1-N NaCl/EG solution driven under similar conditions. 
initial value of $R_{0}$ equal to $22 \mu \mathrm{m}$. However, as we have previously mentioned, the numerical computations were done with other values of the initial bubble radius ranging from $13 \mu \mathrm{m}$ to $26 \mu \mathrm{m}$, and the corresponding optical pulse widths are found to vary from about $30 \mathrm{~ns}$ to $80 \mathrm{~ns}$. In addition, the predicted optical pulse widths are found to reduce with an increase in the acoustic pressure amplitude and this variation is within $5 \mathrm{~ns}$ for a change of acoustic pressure amplitude from 0.93 bar to 1.1 bar [53]. These predictions suggest that the optical pulse width is very sensitively dependent on the initial bubble radius and to a lesser extent on the acoustic pressure amplitude within the range considered presently. The numerically obtained large variation of the optical pulse width with a change in the initial bubble radius indicates that, in the experiments also, the initial radius may not be the same under the presently observed SL conditions, since, as indicated, for example, in Fig. 4, there is a large variation in the observed optical pulse widths also.

\section{Effects of gas thermal conductivity and comparison with experimental results}

The numerical results presented certainly predict the large pulse widths observed experimentally and also provide an explanation for the large variation on the basis of the sensitivity of the pulse width to certain physical parameters such as the initial bubble radius. Experimental observations, however, as indicated in Table I clearly show a systematic variation of the pulse width with a change of dissolved gas. We have examined whether this trend could also be predicted using the formulation presented. For an initial radius of $22 \mu \mathrm{m}$ and an acoustic pressure amplitude of $0.93 \mathrm{bar}$, our numerical results with argon gas predict a pulse width of 62 ns, which is in good agreement with the experimentally observed most probable value of around $62 \mathrm{~ns}$ (this is expected since some parameters were fixed to obtain the agreement). Keeping the same initial conditions, namely $R_{0}=22 \mu \mathrm{m}$, $P_{a}=0.93$ bar, and $n_{0}=1.1 \times 10^{10}$, numerical computations along the same lines as argon were done for krypton and helium. The conductivity dependence on temperature for these two gases was also taken into account on the basis of the data provided in Refs. [47,48]. The details of the computation are again provided in [32], and here we provide only the final results. In Fig. 10, the synthetic optical pulse shapes for the three noble gases are presented. It is clear that as the gas thermal conductivity increases, the optical pulse full width at half maximum (FWHM) decreases. For example, for krypton, which has the lowest thermal conductivity, the predicted pulse width is $94 \mathrm{~ns}$, whereas for helium, which has the highest thermal conductivity, it is $22 \mathrm{~ns}$; as indicated earlier, it is $62 \mathrm{~ns}$ for argon. These predictions can be compared with the observations, and as indicated in Table I, the most probable optical pulse widths for $\mathrm{Kr}$, Ar, and $\mathrm{He}$ are 82 ns, $62 \mathrm{~ns}$, and $42 \mathrm{~ns}$, respectively. In general, the agreement can be considered to be quite satisfactory. In addition, from Fig. 10, we note that the pulse height for helium is significantly smaller than that for krypton. This is consistent with the experimental findings, in the sense that the maximum value of the pulse area for helium is almost half that for

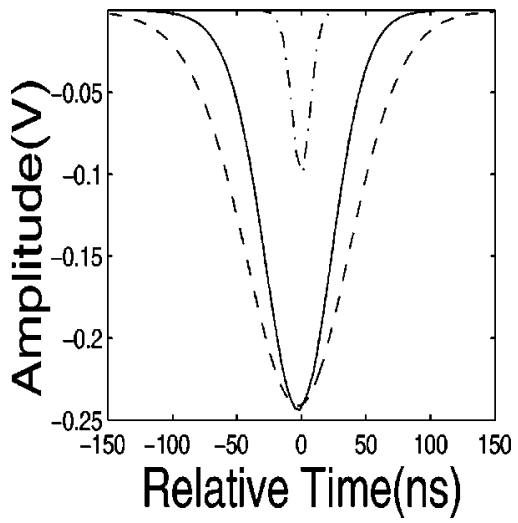

FIG. 10. The synthetic SL optical pulses as a function of relative time for $22 \mu \mathrm{m}$ krypton (--- line), argon ( - line), and helium $(-\cdot-$ line $)$ bubbles in glycol; $P_{a}=0.93$ bar and $f_{a}=32 \mathrm{kHz}$.

krypton (see Table I). Therefore, the numerical computations based on the mathematical formulation of Prosperetti et al. [43] are able to predict many of the important experimental observations involving SL in the form of resonance radiation. In particular, the generally good agreement of the synthetic optical pulse shape with the experimental data is satisfying, however we do need to consider the precise link between the optical pulse widths and the noble gas thermal conductivity, and this is done below.

Our spectra measurements as reported in [32] show that the only emission present with all three noble gases used is the sodium-D line emission. We did not find any evidence of other possible emissions like that from the noble gases themselves or from the vapor (or its products) of the liquid used. Extraordinary care was taken to remove all the air and hence there was no continuum observed even if a small amount of air was left over; in particular, this was the case at high drive levels. In view of this, as indicated earlier, the modeling of the presently observed SL is on the basis of computing the number of excited sodium atoms, and the key variable from the Boltzmann relationship is the temperature. Therefore, the optical pulse characteristics can be directly related to the magnitude and duration of the high temperatures reached within the bubble, and the relationship of this to the thermal conductivity of the gas has been the subject of various investigations $[6,21,28,35,54]$. An increase in the peak temperature inside a cavitation bubble in the order of noble gases from helium to xenon has recently been inferred on the basis of spectral studies [6]. Similarly, our numerical simulations also show that the temporal behavior of the temperatures within the bubble is strongly influenced by the thermal conductivity of the gas; that is not only the magnitudes decrease as we go from krypton to helium, but also the FWHM decreases [32]. Vuong and Szeri [55] have indicated that both the magnitude and the duration of the peak temperature at the origin of a collapsing bubble are not only influenced by the gas thermal conductivity but also by other factors such as the molecular mass. However, their conclusions are for a strongly forced bubble where the wavy nature of the gas dynamics inside the bubble dominates and may not be relevant in our case of a mildly forced bubble, in which case the pressure is assumed to be spatially uniform. Therefore, in the 
present case, we can directly link the decrease in the optical pulse width with an increase in the gas thermal conductivity through its effect on the temperature field.

\section{CONCLUDING REMARKS}

In the SL regime examined by us, only a single bubble going through an unstable motion participates in the SL process. It is possible that a search in the parameter space may enable conditions to be found for establishing stable singlebubble SL (SBSL) with narrowband emission. A related issue is how the species responsible for the narrowband emission (in the present experiments, sodium atoms) can find their way inside a bubble going through a repetitive motion associated with stable SBSL. This point assumes significance since in the present study, a best fit to a synthetic optical pulse with one set of experimental data yields a value for the number of sodium atoms in the bubble to be $1.1 \times 10^{10}$; this corresponds to an atomic density of $2.5 \times 10^{17}$ atoms per $\mathrm{cm}^{3}$ for a $22-\mu \mathrm{m}$ bubble. This value being rather high, one wonders as suggested by some authors earlier [23] whether the sodium atoms find their way inside the bubble through liquid droplet injection by surface instability or jet formation at the last stages of bubble collapse.

The generally good agreement found between the predictions using a SL model based on the bubble dynamics approach of KPE [28] and the experimental observation clearly gives strong support to our earlier hypothesis that the SL in the form of sodium-D line resonance radiation is indeed associated with "soft" bubble collapse. This raises interesting possibilities of controlled "cavitation bubble collapse activity,' which may result in significantly more efficient sonochemical reactions. Presently this aspect has not been considered in the design of sonochemical reactors. The most commonly used device, namely the ultrasonic horn, may induce cavitation fields that produce unwanted products.

\section{ACKNOWLEDGMENT}

This work has been supported by a CSIR Extramural Research Grant.
[1] A. J. Walton and G. T. Reynolds, Adv. Phys. 33, 595 (1984).

[2] L. A. Crum, Phys. Today 47(9), 22 (1994).

[3] T. J. Matula, Philos. Trans. R. Soc. London, Ser. A 375, 225 (1999).

[4] R. E. Verrall and C. M. Sehgal, in Ultrasound: Chemical, Physical, and Biological Effects, edited by K. S. Suslick (VCH, New York, 1988), p. 227.

[5] I. E. El'pinear, Ultrasound: Physical Chemical and Biological Effects (Consultant Bureau, New York, 1964), Chap. 3.

[6] B. W. McNamaraIII, Y. T. Didenko, and K. S. Suslick, Nature (London) 401, 772 (1999); Y. T. Didenko, W. B. McNamara III, and K. S. Suslick, Phys. Rev. Lett. 84, 777 (2000).

[7] H. G. Flynn, in Physics of Acoustic Cavitation in Liquids, Physical Acoustics Principle and Methods 1B, edited by W. P. Mason (Academic, New York, 1964).

[8] M. S. Plesset and A. Prosperetti, Annu. Rev. Fluid Mech. 9, 145 (1977).

[9] C. E. Brennen, Cavitation and Bubble Dynamics (Oxford University Press, New York, 1995).

[10] D. F. Gaitan, Ph.D. thesis, University of Mississippi (1990).

[11] D. F. Gaitan et al., J. Acoust. Soc. Am. 91, 3166 (1992).

[12] B. P. Barber et al., Phys. Rep. 281, 65 (1997).

[13] S. J. Putterman, Sci. Am. 272, 32 (1995).

[14] S. J. Putterman and K. R. Weninger, Annu. Rev. Fluid Mech. 32, 445 (2000).

[15] S. Hilgenfeldt and D. Lohse, Curr. Sci. 78, 238 (2000).

[16] B. P. Barber and S. J. Putterman, Nature (London) 352, 318 (1991).

[17] B. Gompf et al., Phys. Rev. Lett. 79, 1405 (1997); R. A. Hiller, S. J. Putterman, and K. R. Weninger, ibid. 80, 1090 (1998).

[18] M. J. Moran and D. Sweider, Phys. Rev. Lett. 80, 4987 (1998); R. Pecha et al., ibid. 81, 717 (1998).

[19] R. Hiller, S. J. Putterman, and B. P. Barber, Phys. Rev. Lett. 69, 1182 (1992).
[20] R. G. Holt and D. F. Gaitan, Phys. Rev. Lett. 77, 3791 (1996).

[21] S. Hilgenfeldt, S. Grossmann, and D. Lohse, Nature (London) 398, 402 (1999); S. Hilgenfeldt, S. Grossman, and D. Lohse, Phys. Fluids 11, 1318 (1999).

[22] W. C. Moss et al., Phys. Rev. E 59, 2986 (1999).

[23] T. J. Matula et al., Phys. Rev. Lett. 75, 2602 (1995).

[24] K. Yasui, Phys. Rev. Lett. 83, 4297 (1999).

[25] Y. T. Didenko and T. V. Gordeychuk, Phys. Rev. Lett. 84, 5640 (2000).

[26] T. J. Matula, R. A. Roy, and P. D. Mourad, J. Acoust. Soc. Am. 101, 1994 (1997).

[27] S. Hilgenfeldt et al., J. Fluid Mech. 365, 171 (1998).

[28] V. Kamath, A. Prosperetti and F. N. Egolfopoulos, J. Acoust. Soc. Am. 94, 248 (1993).

[29] K. S. Suslick et al., Philos. Trans. R. Soc. London, Ser. A 357, 335 (1999).

[30] A. Giri and V. H. Arakeri, Phys. Rev. E 58, R2713 (1998).

[31] 98\% purity; some limited experiments were done with higher purity but there was no discernible effect.

[32] A. Giri, Ph.D. thesis, Indian Institute of Science, Bangalore, India (2000).

[33] A. T. Ellis, J. Acoust. Soc. Am. 27, 913 (1955).

[34] P. K. Chendke and H. S. Fogler, J. Phys. Chem. 87, 1362 (1983); P. K. Chendke and H. S. Fogler, ibid. 87, 1644 (1983); E. B. Flint and K. S. Suslick, J. Am. Chem. Soc. 111, 6987 (1989); D. W. Kuhns, A. M. Brodsky, and L. W. Burgess, Phys. Rev. E 57, 1702 (1998).

[35] E. B. Flint and K. S. Suslick, J. Phys. Chem. 95, 1484 (1991).

[36] J. A. Ketterling and R. E. Apfel, Phys. Rev. Lett. 81, 4991 (1998).

[37] T. R. Stottlemyer and R. E. Apfel, J. Acoust. Soc. Am. 102, 1418 (1997); J. Holzfuss, M. Rüggeberg, and A. Billo, Phys. Rev. Lett. 81, 5434 (1998).

[38] D. F. Gaitan and R. G. Holt, Phys. Rev. E 59, 5495 (1999). 
[39] S. Hilgenfeldt, D. Lohse, and M. P. Brenner, Phys. Fluids 8, 2808 (1996).

[40] A. Prosperetti and Y. Hao, Philos. Trans. R. Soc. London, Ser. A 357, 203 (1999).

[41] R. Holt et al., Phys. Rev. Lett. 72, 1376 (1994), have pointed out that pulse-to-pulse variation is also observed with SBSL when the system is not driven at optimum conditions; they observe complex temporal behavior of SBSL flashes. Of course, when the system is driven at optimum conditions, remarkable periodicity of the SBSL phenomenon is present [16]. In our case, we do not expect the controlling parameters to be identical for each SL flash, and in this sense, the temporal behavior would be complex, as found by Holt et al. (1994).

[42] On the basis of an important study by D. Lohse et al., Phys. Rev. Lett. 78, 1359 (1997), the question of whether any $\mathrm{N}_{2}$ is left in the bubble needs to be examined. Under our experimental conditions as indicated in Fig. 7, our numerical simulations indicate the maximum bubble temperature to be about $2800 \mathrm{~K}$ and this temperature is significantly lower than the dissociation temperature of $\mathrm{N}_{2}(\sim 9000 \mathrm{~K})$. Hence, the fraction of $\mathrm{N}_{2}$ that will dissociate would be very small. In addition, the combination of the fact that we have no $\mathrm{O}_{2}$ present and also the vapor pressure of the liquid used (ethylene glycol) is very small $(\sim 0.009$ Torr $)$ prevents the possibility of products such as $\mathrm{NO}_{2}$ and $\mathrm{NO}$ being formed in abundance. Therefore, we anticipate that, unlike in the case of SBSL, most of the $\mathrm{N}_{2}$ will remain in the bubble without "burning off' or dissociation.

[43] A. Prosperetti, L. Crum, and W. Commander, J. Acoust. Soc. Am. 83, 502 (1988).

[44] J. B. Keller and M. J. Miksis, J. Acoust. Soc. Am. 68, 628 (1980).

[45] Actually, the proper boundary conditions at the bubble interface should be the temperature continuity and the heat flux continuity between the liquid and the gas. In that case, one has to solve the conservation equation of energy in the liquid also.
However, Kamath et al. [28] show that the temperature rise at the surface of a bubble is around $40 \mathrm{~K}$ during the bubble collapse phase under a moderate forcing when water is the host liquid. On this basis, the present computations are based on using $T=T_{\infty}$ at $r=R$.

[46] G. A. Cook, Argon, Helium and the Rare Gases (Interscience, New York, 1961), Vol. I.

[47] D. J. Collins and W. A. Menard, ASME J. Heat Transfer 88, 52 (1966).

[48] D. J. Collins, R. Greif, and A. E. Bryson Jr., Int. J. Heat Mass Transf. 8, 1209 (1965).

[49] One may question the equilibrium assumption, in particular in view of the time scales involved; however, in [6] experimental evidence has been provided to show that line emissions during bubble collapse can be considered to be thermally equilibrated and this may be due to the fact that thermal equilibrium is achieved from atomic and molecular collisions that involve time scales in the picosecond regime.

[50] C. Th. J. Alkemade and R. Herrmann, Fundamentals of Analytical Flame Spectroscopy (Adam Hilger Ltd., Bristol, 1979), pp. $105-108$.

[51] B. P. Barber, Ph.D. thesis, University of California, Los Angeles, 1992 (unpublished).

[52] K. J. Taylor and P. D. Jarman, Aust. J. Phys. 23, 319 (1970).

[53] The prediction that the optical pulse widths reduce with increased drive pressure contradicts what is observed in the case of SBSL (Hilgenfeldt et al. [21]). This is probably related to the fact that in the present numerical computations, we assumed that the $R_{0}$ value does not change, whereas it is expected to change as the drive pressure changes. A model that includes the change in $R_{0}$ as $P_{a}$ changes [21] is likely to show results that are consistent with the SBSL findings.

[54] W. C. Moss, D. B. Clarke, and D. A. Young, Science 276, 1398 (1997).

[55] V. Q. Vuong and A. J. Szeri, Phys. Fluids 8, 2354 (1996). 